\title{
Buffer-Aided Relaying for Two-Hop Secure Communication
}

\author{
Jing Huang, Member, IEEE, and A. Lee Swindlehurst, Fellow, IEEE
}

\begin{abstract}
We consider using a buffer-aided relay to enhance security for two-hop half-duplex relay networks with an external eavesdropper. We propose a link selection scheme that adapts reception and transmission time slots based on channel quality, while considering both the two-hop transmission efficiency and security. Closed-form expressions for the secrecy throughput and the secrecy outage probability (SOP) are derived, and the selection parameters are optimized to maximize the secrecy throughput or minimize the SOP. We then analyze two sub-optimal link selection schemes that in general only require a line search to solve the optimization problem, and we show that, under certain conditions, these approaches also admit closed-form solutions. All schemes are discussed in the context of two different scenarios where the relay either knows or does not know the channel to the legitimate receiver. In the former case, the relay adopts adaptive-rate transmission, whereas for the latter, it uses fixed-rate transmission. Numerical results show that buffer-aided relaying provides a significant improvement in security compared with conventional unbuffered relaying. Furthermore, the performance of the suboptimal schemes is shown to approach the optimal one for certain ranges of signal-to-noise-ratio (SNR) or SOP constraints.
\end{abstract}

Index Terms-Buffered relay, wiretap channel, physical layer security, outage probability, relay networks.

\section{INTRODUCTION}

A MONG various advanced wireless network architectures studied in the literature, two-hop relay-based networks have received considerable research attention due to their advantages of improving network throughput and enlarging transmission coverage [1], [2]. As an extension to traditional multi-antenna systems, this type of cooperative communication can provide additional degrees of freedom or diversity gains with relatively flexible node deployment. However, relay networks also have to face security issues at the physical layer, since they extend the area over which the private transmitted signals may be intercepted by unintended receivers or eavesdroppers. Therefore, there have been intensive efforts aimed at improving physical layer security for relay networks [3]-[18].

Manuscript received February 2, 2014; revised May 7, 2014; accepted June 23, 2014. Date of publication July 1, 2014; date of current version January 7, 2015. This work was supported in part by the U.S. Army Research Office MURI under Grant W911NF-07-1-0318 and in part by the U.S. National Science Foundation under Grant CCF-1117983. The paper was presented in part at Asilomar 2013. The associate editor coordinating the review of this paper and approving it for publication was W. Zhang.

J. Huang is with Qualcomm Technologies Inc., Santa Clara, CA 95051 USA (e-mail: jinghuang@qti.qualcomm.com).

A. L. Swindlehurst is with the Center for Pervasive Communications and Computing, University of California, Irvine, CA 92697 USA (e-mail: swindle@uci.edu).

Color versions of one or more of the figures in this paper are available online at http://ieeexplore.iee.org.

Digital Object Identifier 10.1109/TWC.2014.2334602
In research on relay networks with secrecy constraints, the relays can be categorized according to their functionality. In addition to acting as pure relays [4], [5], they can serve as jammers or helpers that cooperatively transmit artificial noise to confuse the eavesdropper [6]-[8]. Relays can also play dual roles of simultaneously forwarding information and transmitting artificial noise [9]-[11]. The authors in [9] propose a relay selection scheme where one relay is chosen to forward a message and a second relay is chosen to perform jamming against the eavesdropper. Ding et al. [10] investigate a case where a group of relays are formed to transmit a jamming signal in the null space of the legitimate receiver. In [11], a cooperative jamming scheme is proposed for the two-hop multiple-input multiple-output (MIMO) relay channel in which the relay can split its power between information and jamming signals. More recently, there has been research conducted on scenarios with untrusted relays, where the relay is in effect also an eavesdropper, even though it complies with the source's request to forward messages to the destination [15]-[18].

The relays considered in most of the previous work on halfduplex relay-eavesdropper channels are assumed to receive the signal in one time slot and forward it in the following one. This fixed-schedule two-hop protocol, albeit relatively simple, has limitations on transmission efficiency and diversity performance. Recently, buffer-aided relays have been studied for conventional relay networks without secrecy concerns for increased flexibility [19]-[23]. While [19], [20] still consider a fixed two-hop transmission schedule, [21]-[23] assume the relay can adapt reception and transmission time slots based on the quality of the source-relay and relay-destination channels. Zlatanov et al. [21] show that a buffer-aided relay can provide both throughput and diversity gain by adaptive link selection. In such cases, transmission efficiency can be improved by choosing to let the source transmit in the first hop, and then store the data in the relay's buffer if the second-hop channel is weak. The relay delays transmission until the quality of the secondhop channel sufficiently improves. This motivates the use of buffered relaying in the relay-eavesdropper channel, since this relaying protocol can not only improve two-hop efficiency, but also enable an on-off transmission strategy [24], [25] which allows the transmitter to delay its signal if the legitimate link is relatively weak. This is related to the observation in [25] which shows that the secrecy outage probability (SOP) can be lowered by conditioning on the event of actual message transmission.

In this paper, we study a two-hop decode-and-forward (DF) relay channel with an external eavesdropper, where the relay is aided by a buffer and therefore can adaptively choose to either transmit or receive in each time slot. We propose a 


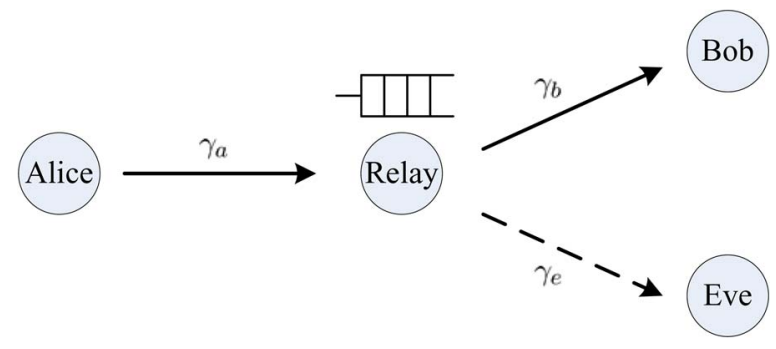

Fig. 1. Illustration of the system model: A source (Alice) communicates with a destination (Bob) via a half-duplex buffered relay, with the relay being wiretapped by an eavesdropper (Eve).

link selection scheme that considers both two-hop transmission efficiency and secrecy constraints to determine which node should transmit in each time slot. We then derive closed-form expressions for the secrecy throughput and the SOP, which we can use to optimize the link selection parameters for two different problems: 1) maximizing the secrecy throughput under a desired SOP constraint, and 2) minimizing the SOP under a desired secrecy throughput threshold. We also investigate two sub-optimal complexity-reduced link selection policies directly induced from the optimal scheme, and show that their performance approaches the optimal one for certain ranges of signal-to-noise-ratio (SNR) or SOP constraint. In addition, we consider the impact of the availability of transmit channel state information (CSI) at the relay, where the relay may adopt either adaptive-rate or fixed-rate transmission to the receiver. Furthermore, we propose a simple approach for modifying the link selection policies when delay constraints are present or the buffer is of limited size. The proposed schemes are shown to provide significant gains in security performance compared to conventional unbuffered relaying. Note that a recent work [26] has investigated the relay selection schemes and corresponding SOP performance for secure buffer-aided relay networks, while on the other hand our work focuses more on single relay channels and considers the trade-off between secrecy throughput and SOP.

The remainder of this work is organized as follows. The mathematical model of the buffer-aided relaying protocol is introduced in Section II. The optimal link selection policy for adaptive-rate relaying is proposed in Section III, and expressions for the corresponding secrecy throughput and SOP are derived. Two sub-optimal link selection schemes are discussed in Section IV, and the scenario of fixed-rate relaying with link selection is investigated in Section V. Selected numerical results are shown in Section VII, and we conclude in Section VIII.

\section{MAthematical Model}

We consider a half-duplex two-hop relaying network composed of a source (Alice), a destination (Bob), and a DF relay equipped with a buffer, as shown in Fig. 1. Each node is assumed to have a single antenna. The DF relay is able to store the decoded data packets received from Alice before forwarding them to Bob. Each transmission occurs in equal-length time slots. In order to focus on the impact of link selection policies on the security performance, we assume that Alice and the relay are located in one cluster, while Bob and Eve are located in another [8], [9], [27], in which case there is no direct link for the Alice-Bob and Alice-Eve channels, and communication can only rely on the relay. The channel is assumed to be stationary and ergodic with frequency non-selective Rayleigh block fading, i.e. the channel gains remain constant during one time slot, but change independently from one time slot to the next. We also assume Alice and the relay transmit with a fixed power $P_{a}$ and $P_{r}$, respectively.

In the $k$-th time slot, the complex channel gains of each link are denoted as $h_{i, k}$ where $i \in\{a, b, e\}$ represents which of the three nodes Alice, Bob and Eve is respectively involved. The noise variances at the relay, Bob, and Eve are denoted by $\sigma_{r}^{2}$, $\sigma_{b}^{2}$ and $\sigma_{e}^{2}$ respectively. The instantaneous SNRs for each link are then given by $\gamma_{a, k}=P_{a}\left|h_{a, k}\right|^{2} / \sigma_{r}^{2}, \gamma_{b, k}=P_{r}\left|h_{b, k}\right|^{2} / \sigma_{b}^{2}$ and $\gamma_{e, k}=P_{r}\left|h_{e, k}\right|^{2} / \sigma_{e}^{2}$ as labeled in Fig. 1. Thus, each SNR $\gamma_{i, k}$ is exponentially distributed with hazard rate $1 / \bar{\gamma}_{i}$ with probability density function (p.d.f.) given by

$$
f_{i, k}(x)=\frac{1}{\bar{\gamma}_{i}} e^{-\frac{x}{\bar{\gamma}_{i}}}, \quad x \geq 0, \quad i \in\{a, b, e\} .
$$

We assume that Alice knows the relay's CSI, and the relay may or may not know Bob's CSI, but neither of them knows the instantaneous CSI of Eve. In each time slot, due to the halfduplex constraint, either Alice or the relay will be selected for transmission. The link selection decision depends on the channel conditions, and the optimization of the decision parameters will be discussed in the following sections.

For a given time slot $k$, if a transmission occurs in the first hop, Alice will adaptively adjust her transmission rate $R_{a, k}$ to be arbitrarily close to the capacity, i.e., $R_{a, k}=C_{a, k}=$ $\log _{2}\left(1+\gamma_{a, k}\right)$, such that no outage events occur in the first hop. If the second-hop link is selected for transmission, the relay will forward the secret messages decoded and stored in its buffer during the first hop. We assume the relay uses a codebook $\mathcal{C}\left(2^{n R_{b, k}}, 2^{n R_{s}^{\prime}}, n\right)$ where $R_{s}^{\prime}$ is the intended secrecy rate, $n$ is the codeword length, $2^{n R_{b, k}}$ is the size of the codebook, and $2^{n R_{s}^{\prime}}$ is the number of confidential messages to send. The $2^{n R_{b, k}}$ codewords are randomly grouped into $2^{n R_{s}^{\prime}}$ bins. To send confidential message $w \in\left\{1, \ldots, 2^{n R_{s}^{\prime}}\right\}$, the relay will use a stochastic encoder to randomly select a codeword from bin $w$ and send it over the channel. If Bob's CSI is available at the relay, the encoder will adaptively set $R_{b, k}$ to be arbitrarily close to the channel capacity from the relay to Bob, i.e. $R_{b, k}=C_{b, k}=\log _{2}\left(1+\gamma_{b, k}\right)$. Otherwise, a fixed $R_{b, k}$ will be set during the transmission, as explained later.

Since the instantaneous CSI for Eve is not available at the relay, we assume the encoder will set a fixed value for the intended positive secrecy rate $R_{s}$, and thus $R_{s}^{\prime}=\min \left\{R_{s}, Q_{k}\right\}$ where $Q_{k}$ represents the number of bits in the buffer at the beginning of the $k$-th time slot. If $R_{s}>Q_{k}$, dummy bits or zero padding can be used in order to maintain a fixed rate $R_{s}$. As a result, a secrecy outage event [28] may occur in the transmission, and we will analyze the probability of such outage events in the following sections. We also assume that Alice always has data to transmit and the buffer at the relay is large enough to avoid overflow. 
Remark 1: When Eve's instantaneous CSI is not available, an adaptive encoder with varying secrecy rate $R_{s}$ based on Bob's CSI may yield higher secrecy throughput [29], but it would require an online optimization for each channel realization and hence significantly higher computational complexity. Therefore, we only focus on fixed $R_{s}$ in this paper.

Remark 2: The buffer-aided relay model in this paper is similar to a buffer-aided transmitter with a given data arrival process [30]-[33], but they differ in the following important aspects: 1) the mean arrival rate in a buffer-aid relay system is a function of the parameters of the chosen transmission scheme, while in a buffer-aided transmitter system, the mean arrival rate is a constant independent of the transmission scheme; 2) a buffer-aided relay operates in a half-duplex channel where the buffer does not accumulate data during transmission (i.e., the buffer must choose to either listen or transmit), while data in a buffer-aided transmitter can arrive continuously. Thus the problem formulation and solution will be different for the two cases.

\section{Link SELECTION DESIGN FOR BUfFERED ADAPTIVE-RATE RELAYING}

In this section, we propose a link selection policy aimed at maximizing the secrecy throughput under desired SOP constraints, or minimizing the SOP under certain throughput requirements. We focus here on the case where the CSI at Bob is available at the relay and thus the relay uses adaptive-rate transmission.

\section{A. Link Selection Policy}

The relay decides whether she should transmit to Bob or listen to Alice during each time slot. The selection should consider the following two aspects: 1) two-hop transmission efficiency - for DF relaying without a direct connection, if the first-hop channel is significantly better than the second-hop, Alice should transmit and the relay should listen and store the decoded data in her buffer. Otherwise, the relay should be selected to forward data to Bob as long as the following condition in (2) is also satisfied: 2) security performance-the channel from the relay to Bob should be strong enough to guarantee a certain SOP. Based on the above considerations and the assumption that the instantaneous CSI of Eve is not available, we propose the following link selection criterion:

$$
I_{k \in\{1, \ldots, N\}}= \begin{cases}1 & \gamma_{b, k} \geq \max \left\{\alpha \gamma_{a, k}, \beta\right\} \\ 0 & \text { otherwise }\end{cases}
$$

where $I_{k}$ is a binary-valued variable with $I_{k}=1 \quad\left(I_{k}=0\right)$ indicating the relay transmits (listens) in the $k$-th time slot, and $\{\alpha, \beta\}$ are two non-negative scalars that determine the threshold for choosing when the relay transmits. The condition $\gamma_{b, k} \geq \alpha \gamma_{a, k}$ relates the relative quality of the links from the relay to Bob and Alice (and hence is important for maintaining the two-hop transmission efficiency), while $\gamma_{b, k} \geq \beta$ is used to assess the quality of the link to Bob in order to provide good secrecy performance. Note that both conditions must be satisfied for the relay to transmit in time slot $k$. The parameters $\alpha$ and $\beta$ must be chosen to optimize the desired performance metric.

The transmission probability of the relay is a function of $\alpha$ and $\beta$, and can be calculated as

$$
\begin{aligned}
p_{b}(\alpha, \beta)= & \operatorname{Pr}\left[\gamma_{b, k} \geq \max \left(\alpha \gamma_{a, k}, \beta\right)\right] \\
= & \operatorname{Pr}\left(\gamma_{b, k} \geq \alpha \gamma_{a, k}, \alpha \gamma_{a, k} \geq \beta\right) \\
& +\operatorname{Pr}\left(\gamma_{b, k} \geq \beta, \alpha \gamma_{a, k}<\beta\right) \\
= & \operatorname{Pr}\left(\beta \leq \alpha \gamma_{a, k} \leq \gamma_{b, k}\right) \\
& +\operatorname{Pr}\left(\gamma_{b, k} \geq \beta\right) \operatorname{Pr}\left(\alpha \gamma_{a, k}<\beta\right) \\
= & e^{-\frac{\beta}{\bar{\gamma}_{b}}}-\frac{\alpha \bar{\gamma}_{a}}{\bar{\gamma}_{b}+\alpha \bar{\gamma}_{a}} e^{-\left(\frac{\beta}{\bar{\gamma}_{b}}+\frac{\beta}{\alpha \bar{\gamma}_{a}}\right)} .
\end{aligned}
$$

\section{B. Secrecy Throughput}

According to the transmission model in Section II and (2), the average arrival rate at the relay buffer can be written as

$$
R_{\text {in }}=\lim _{N \rightarrow \infty} \frac{1}{N} \sum_{k=1}^{N}\left(1-I_{k}\right) R_{a, k}
$$

and the departure secrecy rate considering the queue of the buffer is given by

$$
R_{\text {out }}=\lim _{N \rightarrow \infty} \frac{1}{N} \sum_{k=1}^{N} I_{k} \min \left\{R_{s}, Q_{k}\right\},
$$

which is also the secrecy throughput of the channel. Note that $R_{\text {in }} \geq R_{\text {out }}$ is always valid due to the buffered relaying protocol, and when $R_{\text {in }}>R_{\text {out }}$, the queue of the buffer is said to be in the absorbing state.

Lemma 1: Under a link selection policy that maximizes the throughput, the queue in the buffer is at the edge of nonabsorption where

$$
\lim _{N \rightarrow \infty} \frac{1}{N} \sum_{k=1}^{N} I_{k} \min \left\{R_{s}, Q_{k}\right\}=\lim _{N \rightarrow \infty} \frac{1}{N} \sum_{k=1}^{N} I_{k} R_{s} .
$$

Therefore, assuming that $\gamma_{a, k}$ and $\gamma_{b, k}$ are ergodic and stationary random processes, then

$$
\mathbb{E}\left\{\left(1-I_{k}\right) R_{a, k}\right\}=\mathbb{E}\left\{I_{k} R_{s}\right\}
$$

and the corresponding throughput is

$$
R_{t}=\mathbb{E}\left\{I_{k} R_{s}\right\}
$$

Proof: The proof is similar to that for Theorems 1 and 2 in [23], so here we provide just a brief explanation. Denote the sets of indices $k$ with $I_{k}=1$ by $\mathcal{S}$ (time slots when the relay transmits) and $I_{k}=0$ by $\overline{\mathcal{S}}$. Assuming a link selection policy that results in $R_{\text {in }}>R_{\text {out }}$, one can always improve the policy by moving some of the indices from $\overline{\mathcal{S}}$ to $\mathcal{S}$ to increase the throughput until it reaches the point where $R_{\text {in }}=R_{\text {out }}$. After this point, further movement of the indices will cause both $R_{\text {in }}$ and $R_{\text {out }}$ to decrease. Therefore, under the optimal policy, the queue stays at the edge of non-absorption where 
we have $R_{\text {in }}=R_{\text {out }} \leq \lim _{N \rightarrow \infty}(1 / N) \sum_{k=1}^{N} I_{k} R_{s}$. We can easily show that $R_{\text {out }}<\lim _{N \rightarrow \infty}(1 / N) \sum_{k=1}^{N} I_{k} R_{s}$ does not hold since otherwise the transition from absorbing to nonabsorbing state is discontinuous, which is not possible due to the fact that $\lim _{N \rightarrow \infty} R_{a, k} / N \rightarrow 0$ and $\lim _{N \rightarrow \infty} R_{s} / N \rightarrow 0$. Therefore, $R_{\text {in }}=R_{\text {out }}=\lim _{N \rightarrow \infty}(1 / N) \sum_{k=1}^{N} I_{k} R_{s}$ holds at the optimal point and (6) is then inferred due to the ergodicity of $\gamma_{a, k}$ and $\gamma_{b, k}$.

Lemma 1 indicates that under the optimal link selection policy, the impact of the event where $R_{s}>Q_{k}, k \in\{1, \ldots, N\}$ is negligible, and the throughput maximization can be formulated based on (7) with the rate balance constraint (6). Since $R_{s}$ is a fixed scalar, the throughput is

$$
R_{t}=\mathbb{E}\left\{I_{k} R_{s}\right\}=p_{b} R_{s}
$$

where $p_{b}$ is given by (3).

Since the statistics of $\gamma_{a, k}, \gamma_{b, k}$ and $\gamma_{e, k}$ are assumed to be time-invariant, we will use $\gamma_{a}=\gamma_{a, k}, \gamma_{b}=\gamma_{b, k}$ and $\gamma_{e}=\gamma_{e, k}$, $k \in\{1, \ldots, N\}$ throughout the remainder of the paper. Next, we calculate the average arrival rate at the relay. According to (2), Alice transmits when $\gamma_{b}<\max \left(\alpha \gamma_{a}, \beta\right)$ which is equivalent to $\left(\gamma_{b}<\beta\right) \cup\left(\beta<\gamma_{b}<\alpha \gamma_{a}\right)$. Therefore, we have the following proposition,

Proposition 1: The average arrival rate, based on the link selection scheme given in (2), can be written as

$$
\begin{aligned}
\mathbb{E}\{ & \left.\left(1-I_{k}\right) R_{a}\right\} \\
= & \int_{0}^{\beta}\left(\int_{0}^{\infty} \log _{2}\left(1+\gamma_{a}\right) f_{a}\left(\gamma_{a}\right) d \gamma_{a}\right) f_{b}\left(\gamma_{b}\right) d \gamma_{b} \\
& +\int_{\beta}^{\infty}\left(\int_{\frac{\gamma_{b}}{\alpha}}^{\infty} \log _{2}\left(1+\gamma_{a}\right) f_{a}\left(\gamma_{a}\right) d \gamma_{a}\right) f_{b}\left(\gamma_{b}\right) d \gamma_{b} \\
= & \log _{2}\left(1+\frac{\beta}{\alpha}\right) \frac{\alpha \bar{\gamma}_{a}}{\alpha \bar{\gamma}_{a}+\bar{\gamma}_{b}} e^{-\left(\frac{\beta}{\alpha \bar{\gamma}_{a}}+\frac{\beta}{\bar{\gamma}_{b}}\right)} \\
& +\frac{1}{\ln 2}\left(1-e^{-\frac{\beta}{\bar{\gamma}_{b}}}\right) e^{\frac{1}{\bar{\gamma}_{a}}} E_{1}\left(\frac{1}{\bar{\gamma}_{a}}\right) \\
& +\frac{1}{\ln 2} e^{\left(\frac{1}{\bar{\gamma}_{a}}-\frac{\beta}{\bar{\gamma}_{b}}\right)} E_{1}\left(\frac{\alpha+\beta}{\alpha \bar{\gamma}_{a}}\right) \\
& -\frac{1}{\ln 2} \frac{\bar{\gamma}_{b}}{\alpha \bar{\gamma}_{a}+\bar{\gamma}_{b}} e^{\frac{1}{\bar{\gamma}_{a}}+\frac{\alpha}{\bar{\gamma}_{b}}} E_{1}\left[\left(\frac{1}{\bar{\gamma}_{a}}+\frac{\alpha}{\bar{\gamma}_{b}}\right)\left(1+\frac{\beta}{\alpha}\right)\right]
\end{aligned}
$$

where $\alpha$ and $\beta$ are non-negative scalars representing the SNR threshold for the link selection policy, and $E_{1}(x)=$ $\int_{x}^{\infty}\left(e^{-t} / t\right) d t, x>0$ is the exponential integral function.

Proof: See Appendix A.

\section{Secrecy Outage Probability}

In addition to secrecy throughput, an important security measurement for our model is the SOP, which determines the likelihood of achieving a certain secrecy rate in fading channels. For single hop transmission, the traditional SOP is defined as $p_{\text {sop }}=\operatorname{Pr}\left\{C_{s}<R_{s}\right\}$ where the secrecy capacity $C_{s}=$ $\max \left\{0, C_{b}-C_{e}\right\}$ depends on the channel capacity at Bob $C_{b}$ and at Eve $C_{e}$ [28]. An alternative expression is given in [34] as $p_{\text {sop }}=\operatorname{Pr}\left\{C_{e}>R_{b}-R_{s}\right\}$, which takes into account the rate of the transmitted codewords. More recently, the SOP conditioned on actual private message transmission is introduced in [25] as $p_{\text {sop }}=\operatorname{Pr}\left\{C_{e}>R_{b}-R_{s} \mid\right.$ message transmission $\}$, which is more suitable to on-off secure transmission, and we will adopt this formulation in our study.

Since $R_{b}$ is assumed to be adaptively adjusted to $C_{b}$, the SOP is written as

$$
\begin{aligned}
& p_{\text {sop }}\left(R_{s}, \alpha, \beta\right) \\
& =\operatorname{Pr}\left[C_{e}>C_{b}-\min \left(R_{s}, Q_{k}\right) \mid \gamma_{b}>\max \left(\alpha \gamma_{a}, \beta\right)\right] \\
& \leq \operatorname{Pr}\left[C_{e}>C_{b}-R_{s} \mid \gamma_{b}>\max \left(\alpha \gamma_{a}, \beta\right)\right] \\
& =\frac{\operatorname{Pr}\left[C_{e}>C_{b}-R_{s}, \gamma_{b}>\max \left(\alpha \gamma_{a}, \beta\right)\right]}{\operatorname{Pr}\left[\gamma_{b}>\max \left(\alpha \gamma_{a}, \beta\right)\right]} \\
& =\frac{\operatorname{Pr}\left[C_{e}>C_{b}-R_{s}, \beta \leq \alpha \gamma_{a}<\gamma_{b}\right]}{\operatorname{Pr}\left[\gamma_{b}>\max \left(\alpha \gamma_{a}, \beta\right)\right]} \\
& \quad+\frac{\operatorname{Pr}\left[C_{e}>C_{b}-R_{s}, \gamma_{b}>\beta, \alpha \gamma_{a}<\beta\right]}{\operatorname{Pr}\left[\gamma_{b}>\max \left(\alpha \gamma_{a}, \beta\right)\right]} \\
& =\frac{\operatorname{Pr}\left[\beta \leq \alpha \gamma_{a}<\gamma_{b}<2^{R_{s}}\left(1+\gamma_{e}\right)-1\right]}{\operatorname{Pr}\left[\gamma_{b}>\max \left(\alpha \gamma_{a}, \beta\right)\right]} \\
& \quad+\frac{\operatorname{Pr}\left[\beta<\gamma_{b}<2^{R_{s}}\left(1+\gamma_{e}\right)-1\right] \operatorname{Pr}\left[\alpha \gamma_{a}<\beta\right]}{\operatorname{Pr}\left[\gamma_{b}>\max \left(\alpha \gamma_{a}, \beta\right)\right]}
\end{aligned}
$$

which is a function of the desired secrecy rate $R_{s}$ and the link selection parameters $\alpha$ and $\beta$. Note that inequality (a) indicates that the result in (10) is an upper bound for the SOP. However, when using the optimal link selection policy (optimizing $\alpha$ and $\beta$ such that (6) is satisfied), the queue of the buffer is at the edge of non-absorption and the probability of the event $R_{s}>Q_{k}$ is negligible. Therefore, the above upper bound is tight. Further manipulations reveal that,

$$
\begin{aligned}
p_{\text {sop }} & \\
= & \frac{\int_{\frac{\beta+1}{2_{s}}-1}^{\infty} \int_{\beta}^{2^{R_{s}}\left(1+\gamma_{e}\right)-1} \int_{\beta / \alpha}^{\gamma_{b} / \alpha} f_{a}\left(\gamma_{a}\right) f_{b}\left(\gamma_{b}\right) f_{e}\left(\gamma_{e}\right) d \gamma_{a} d \gamma_{b} d \gamma_{e}}{p_{b}} \\
& +\frac{\int_{\frac{\beta+1}{2^{R_{s}}}-1}^{\infty} \int_{\beta}^{2^{R_{s}}\left(1+\gamma_{e}\right)-1} f_{b}\left(\gamma_{b}\right) f_{e}\left(\gamma_{e}\right) d \gamma_{b} d \gamma_{e}}{p_{b}} \\
= & \left(\frac{2^{R_{s}} \bar{\gamma}_{e}}{\left(2^{R_{s}} \bar{\gamma}_{e}+\bar{\gamma}_{b}\right)\left(\frac{\alpha \bar{\gamma}_{a}}{\bar{\gamma}_{b}}+\frac{\alpha \bar{\gamma}_{a}}{2_{s}^{R} \bar{\gamma}_{e}}+1\right)} e^{-\left(\frac{\beta}{\alpha \bar{\gamma}_{a}}+\frac{\beta}{\bar{\gamma}_{b}}+\frac{1+\beta 2^{R} R_{s}}{2^{R_{s} \bar{\gamma}_{e}}}\right)}\right. \\
& \left.\left.+\frac{2^{R_{s}} \bar{\gamma}_{e}}{\left(2^{R_{s}} \bar{\gamma}_{e}+\bar{\gamma}_{b}\right)} e^{-\left(\frac{1+\beta-2^{R_{s}}}{2^{R_{s} \gamma_{e}}}+\frac{\beta}{\bar{\gamma}_{b}}\right.}\right)\left(1-e^{-\frac{\beta}{\alpha \bar{\gamma}_{a}}}\right)\right) \frac{1}{p_{b}} .
\end{aligned}
$$

Note that the encoding at the relay requires that $R_{s} \leq C_{b}=$ $\log _{2}\left(1+\gamma_{b}\right)$, thus $\beta \geq 2^{R_{s}}-1$ always holds and the lower limit of the outer integration is non-negative. 


\section{Optimization of Link Selection Parameters (LS-OPT)}

Now we are ready to formulate our optimization problem. We aim to optimize the secrecy rate $R_{s}$ that the relay uses for encoding, and the transmission threshold $\alpha$ and $\beta$ that the relay uses to perform link selection, such that either 1) the secrecy throughput is maximized under a certain SOP constraint, or 2) the SOP is minimized under a certain secrecy throughput requirement. We refer to the former as Problem P1 and the latter as Problem P2 in the sequel.

Problem P1 is formulated as

$$
\begin{aligned}
\mathbf{P 1}: \max _{R_{s}, \alpha, \beta} & p_{b}(\alpha, \beta) R_{s} \\
\text { s.t. } & p_{\text {sop }}\left(R_{s}, \alpha, \beta\right) \leq \eta \\
& \mathbb{E}\left\{\left(1-I_{k}\right) C_{a}\right\}=\mathbb{E}\left\{I_{k} R_{s}\right\} \\
& R_{s}>0, \alpha \geq 0, \beta \geq 2^{R_{s}}-1,
\end{aligned}
$$

where $\eta(\eta \leq 1)$ is the desired maximum SOP, the expression for $(12 b)$ is given in $(11),(12 c)$ is the condition for maximum throughput, and the average rates are given in (8) and (9), respectively.

For Problem P2, we have

$$
\begin{aligned}
\text { P2: } \min _{R_{s}, \alpha, \beta} & p_{\text {sop }}\left(R_{s}, \alpha, \beta\right) \\
\text { s.t. } & p_{b}(\alpha, \beta) R_{s} \geq \mu \\
& \mathbb{E}\left\{\left(1-I_{k}\right) C_{a}\right\}=\mathbb{E}\left\{I_{k} R_{s}\right\} \\
& R_{s}>0, \alpha \geq 0, \beta \geq 2^{R_{s}}-1,
\end{aligned}
$$

where $\mu>0$ is the minimum desired throughput. Closed-form solutions to $\mathrm{P} 1$ and $\mathrm{P} 2$ are in general not possible, and thus a two dimensional search over $R_{s}$ and $\beta$ must be used in order to find the optimal link selection policy. For given $R_{s}$ and $\beta$, the value of $\alpha$ can be obtained numerically by solving (12c) or $(13 \mathrm{c})$. Note that one can also use the property that $p_{\text {sop }}$ is a monotonically decreasing function of $\beta$ to simplify the search algorithm. In the following section, we will study two sub-optimal schemes that in general only require a line search. Conditions under which a closed-from solution can be obtained will also be discussed.

\section{Sub-Optimal Link Selection Policies}

In this section, we consider two sub-optimal link selection policies that only involve a search for one of the parameters, either $\alpha$ or $\beta$. The two algorithms correspond respectively to either letting $\beta=2^{R_{s}}-1$ or $\alpha=0$ in the LS-OPT scheme proposed in the previous section.

\section{A. Link Selection With Two-Hop Condition (LS-TC)}

When the relay only considers two-hop transmission efficiency, i.e. when it only uses $\alpha$ as the threshold parameter in selecting the link, the selection policy can be written as:

$$
I_{k}^{T C}= \begin{cases}1 & \gamma_{b} \geq \max \left\{\alpha \gamma_{a}, 2^{R_{s}}-1\right\} \\ 0 & \text { otherwise. }\end{cases}
$$

This approach reduces to a scheme similar to that used in [23] for conventional buffer-aided relaying, but with two differences: 1) The throughput maximized in [23] is the public data rate to Bob, while the throughput in our work is the average secrecy rate constrained by the SOP, and 2) we have a minimum threshold of $2^{R s}-1$ for $\gamma_{b}$ to ensure a feasible secrecy codebook. Thus, LS-TC corresponds to the traditional link selection policy adopted in buffered relay system, but with a metric based on average secrecy rate. Analysis of this method allows us to examine the performance of traditional schemes in a secrecy setting, compared to other approaches that consider secrecy constraints. For LS-TC, the transmission probability $p_{b}$, the average arrival rate $\mathbb{E}\left\{\left(1-I_{k}\right) C_{a}\right\}$, and the SOP $p_{\text {sop }}$ are given by (3), (9), and (11) with $\beta$ fixed at $2^{R s}-1$. To solve P1 and P2, one only needs to perform a line search over $R_{s}$, and given $R_{s}$ the value of $\alpha$ can be obtained by solving $\mathbb{E}\left\{\left(1-I_{k}\right) C_{a}\right\}=p_{b} R_{s}$. Since this policy does not consider the secrecy constraint, we expect that the performance of LS-TC will converge to that of LS-OPT only when $\eta$ is close to 1, i.e. with a more relaxed SOP constraint, a fact that will be validated later in the simulations of Section VII.

\section{B. Link Selection With Secrecy Condition (LS-SC)}

Alternatively, a link selection scheme that only considers $\beta$ can be expressed as

$$
I_{k}^{S C}= \begin{cases}1 & \gamma_{b} \geq \beta \\ 0 & \text { otherwise. }\end{cases}
$$

This policy not only considers secrecy, but also indirectly considers the two-hop transmission efficiency since the relay only transmits when her channel to Bob is relatively strong. Note that this approach is similar to the on-off transmission scheme in [25], although the case considered therein focuses on a single-hop channel. Since (15) considers $\beta$ only, we will show in the numerical results that the secrecy throughput of this scheme converges to that of LS-OPT only when $\eta$ is relatively low, i.e. with a stricter SOP constraint.

According to (15), the transmission probability of the relay is simply $p_{b}(\beta)=\operatorname{Pr}\left(\gamma_{b} \geq \beta\right)=e^{-\left(\beta / \bar{\gamma}_{b}\right)}$. The SOP in this case is given by

$$
\begin{aligned}
p_{\text {sop }}\left(R_{s}, \beta\right) & =\operatorname{Pr}\left[C_{e}>C_{b}-R_{s} \mid \gamma_{b}>\beta\right] \\
& =\frac{\operatorname{Pr}\left[\beta<\gamma_{b}<2^{R_{s}}\left(1+\gamma_{e}\right)-1\right]}{\operatorname{Pr}\left[\gamma_{b}>\beta\right]} \\
& =\frac{2^{R_{s}} \bar{\gamma}_{e}}{2^{R_{s}} \bar{\gamma}_{e}+\bar{\gamma}_{b}} e^{-\frac{\beta+1-2}{2^{R_{s}} \bar{\gamma}_{e}}},
\end{aligned}
$$

and the average arrival rate is

$$
\begin{aligned}
\mathbb{E} & \left\{\left(1-I_{k}\right) C_{a}\right\} \\
= & \int_{0}^{\beta}\left(\int_{0}^{\infty} \log _{2}\left(1+\gamma_{a}\right) f_{a}\left(\gamma_{a}\right) d \gamma_{a}\right) f_{b}\left(\gamma_{b}\right) d \gamma_{b} \\
= & \frac{1}{\ln 2} e^{\frac{1}{\bar{\gamma}_{a}}}\left(1-e^{-\frac{\beta}{\bar{\gamma}_{b}}}\right) E_{1}\left(\frac{1}{\bar{\gamma}_{a}}\right) .
\end{aligned}
$$


Equating (17) to the average departure rate $p_{b} R_{s}$, we can express $\beta$ as a function of $R_{s}$,

$$
\beta=\bar{\gamma}_{b} \ln \left(1+\frac{R_{s} \ln 2}{e^{1 / \bar{\gamma}_{a}} E_{1}\left(1 / \bar{\gamma}_{a}\right)}\right) .
$$

Using (18) and assuming $\lambda=\ln 2 / e^{1 / \bar{\gamma}_{a}} E_{1}\left(1 / \bar{\gamma}_{a}\right)$, Problems P1 and P2 then reduce to

$$
\begin{aligned}
& \mathbf{P 1}(\mathrm{LS}-\mathrm{SC}): \max _{R_{s}>0} \frac{R_{s}}{1+\lambda R_{s}} \\
& \text { s.t. } p_{\text {sop }}\left(R_{s}\right) \leq \eta \\
& \quad \ln \left(1+\lambda R_{s}\right) \geq\left(2^{R_{s}}-1\right) / \bar{\gamma}_{b},
\end{aligned}
$$

and

$$
\begin{aligned}
\text { P2 }(\text { LS-SC }): & \min _{R_{s}>0} p_{\mathrm{sop}}\left(R_{s}\right) \\
& \text { s.t. } R_{s} \geq \mu /(1-\mu \lambda) \\
& \quad \ln \left(1+\lambda R_{s}\right) \geq\left(2^{R_{s}}-1\right) / \bar{\gamma}_{b},
\end{aligned}
$$

and both can be solved via a one-dimensional search over $R_{s}$.

Remark 3: From (20b), we know that the desired throughput threshold $\mu$ satisfies $\mu \leq 1 / \lambda$. It can also be seen from (19a) that the secrecy throughput can never exceed $1 / \lambda$, which indicates that the upper bound of the throughput depends on the channel condition of the first hop, which is consistent with the law of conservation of flow.

Remark 4: Intuitively, $p_{\text {sop }}$ should be a monotonically increasing function of $R_{s}$ since a higher $R_{s}$ will result in a higher probability of occurrence for the event $C_{e}>C_{b}-R_{s}$. However, this is not necessarily the case in our setting because, as seen in (18), $R_{s}$ and $\beta$ are not independent. The design of $R_{s}$ affects the design of the parameters of the link selection policy that determines both the secrecy throughput and outage performance. In the following proposition, we will provide a closedform solution to $\mathrm{P} 2$ for a certain range of $\mu$, by characterizing the domain where $p_{\text {sop }}$ is a monotonically increasing function of $R_{s}$.

Proposition 2: If $\mu \in\left[\left(R_{l} /\left(1+\lambda R_{l}\right)\right),\left(R_{u} /\left(1+\lambda R_{u}\right)\right)\right]$, the solution to (20) is the lower bound of $R_{s}$, i.e.

$$
R_{s}=\frac{\mu}{1-\mu \lambda},
$$

where $R_{l}=\left[e^{W(\lambda / \ln 2)}-1\right] / \lambda, W(\cdot)$ is the Lambert $\mathrm{W}$ function, and $R_{u}$ is the root of function $h\left(R_{s}\right)=\ln \left(1+\lambda R_{s}\right)-$ $\left(2^{R_{s}}-1\right) / \bar{\gamma}_{b}=0$.

Proof: Inserting (18) into (16), we can write the SOP as

$$
p_{\text {sop }}\left(R_{s}\right)=\frac{2^{R_{s}} \bar{\gamma}_{e}}{2^{R_{s}} \bar{\gamma}_{e}+\bar{\gamma}_{b}} e^{-\frac{2^{-R_{s}}-1+\psi\left(R_{s}\right)}{\bar{\gamma}_{e}}},
$$

where

$$
\psi\left(R_{s}\right)=\bar{\gamma}_{b} 2^{-R_{s}} \ln \left(1+\lambda R_{s}\right) .
$$

One could try to find values of $R_{s}$ for which the derivative of $p_{\text {sop }}\left(R_{s}\right)$ is non-negative, and thus $p_{\text {sop }}$ is increasing over $R_{s}$. However, the expression for $p_{\text {sop }}^{\prime}\left(R_{s}\right)$ is complicated and it is difficult to find the corresponding domain for $R_{s}$. Therefore, we resort to solving for the sufficient condition $\psi^{\prime}\left(R_{s}\right) \leq 0$ under which $p_{\text {sop }}$ is an increasing function of $R_{s}$. By examining $\psi^{\prime}\left(R_{s}\right) \leq 0$, we have

$$
R_{s} \geq R_{l}=\left[e^{W(\lambda / \ln 2)}-1\right] / \lambda,
$$

where $W(\cdot)$ is the Lambert $\mathrm{W}$ function. Observing that the LHS and RHS of (20c) are respectively concave and convex with respect to $R_{s}$, we know that there is a unique positive root $R_{s}=R_{u}$ where $\ln \left(1+\lambda R_{s}\right)=\left(2^{R_{s}}-1\right) / \bar{\gamma}_{b}$ such that $R_{s} \leq$ $R_{u}$ is equivalent to (20c). Therefore, if $R_{s} \in\left[R_{l}, R_{u}\right], p_{\text {sop }}$ is an increasing function of $R_{s}$ and the solution for Problem P2 is $R_{s}=\mu /(1-\mu \lambda)$, or equivalently,

$$
R_{s}=\frac{\mu}{1-\mu \lambda}, \quad \text { if } \mu \in\left[\frac{R_{l}}{1+\lambda R_{l}}, \frac{R_{u}}{1+\lambda R_{u}}\right] .
$$

\section{Link Selection Design for Buffered FIXED-RATE RELAYING}

The previous sections considered the case where the instantaneous CSI to Bob is available at the relay. However, it is also possible that the relay does not know Bob's instantaneous CSI, for example in the scenario where Bob does not send CSI feedback due to system complexity or security concerns. In such cases, instead of adaptively adjusting $R_{b}$ to $C_{b}$, the encoder at the relay will set a fixed value for $R_{b}$ during the transmission. Unlike the previous cases where link selection can be performed at the relay, in this case the decision is made at Bob, who will then notify the relay through a 1 bit feedback channel. Therefore, in addition to $\left\{R_{s}, \alpha, \beta\right\}$, the transmission design now must take into account the parameter $R_{b}$. It is worth noting that in addition to a secrecy outage, the outage event $C_{b}<R_{b}$ may also occur, and thus we need to also consider the reliability outage probability (ROP) in this case. However, if Bob is able to send 1 bit of feedback to the relay by comparing $C_{b}$ and $R_{b}$, a reliability outage can be avoided [25]. In the following, we will study both of these cases separately.

\section{A. Link Selection With Reliability Outage}

The transmission probability $p_{b}$ and the average arrival rate $\mathcal{E}\left\{\left(1-I_{k}\right) C_{a}\right\}$ for this fixed-rate transmission scheme are still the same as (3) and (9) respectively, but the SOP changes to

$$
\begin{aligned}
p_{\text {sop }} & =\operatorname{Pr}\left[C_{e}>R_{b}-R_{s} \mid \gamma_{b}>\max \left(\alpha \gamma_{a}, \beta\right)\right] \\
& =\operatorname{Pr}\left[C_{e}>R_{b}-R_{s}\right] \\
& =e^{-\frac{2^{R_{b}-R_{s}-1}}{\bar{\gamma}_{e}}},
\end{aligned}
$$

which is only a function of $R_{b}$ and $R_{s}$. Denoting the fixedrate secrecy throughput maximization and SOP minimization problems as $\mathrm{PF} 1$ and $\mathrm{PF} 2$ respectively (as counterparts of the adaptive-rate Problems P1 and P2), we first express PF1 as

$$
\text { PF1 : } \max _{R_{b}, R_{s}, \alpha, \beta} p_{b}(\alpha, \beta) R_{s}
$$




$$
\begin{array}{ll}
\text { s.t. } & p_{\text {sop }}\left(R_{b}, R_{s}\right) \leq \eta \\
& p_{\text {rop }}\left(R_{b}\right) \leq \delta \\
& \mathbb{E}\left\{\left(1-I_{k}\right) C_{a}\right\}=\mathbb{E}\left\{I_{k} R_{s}\right\} \\
& R_{b} \geq R_{s}>0 \\
& \alpha \geq 0, \beta \geq 2^{R_{s}}-1
\end{array}
$$

where $p_{\text {sop }}$ is given in (25), $\delta$ is the desired RIO constraint given by

$$
\begin{aligned}
p_{\text {rop }}\left(R_{b}\right) & =\operatorname{Pr}\left\{C_{b}<R_{b} \mid \gamma_{b} \geq \max \left(\alpha \gamma_{a}, \beta\right)\right\} \\
& =\operatorname{Pr}\left\{C_{b}<R_{b}\right\} \\
& =1-e^{-\frac{2^{R_{b}}-1}{\bar{\gamma}_{b}}},
\end{aligned}
$$

and thus (26c) implies $R_{b} \leq \log _{2}\left(1-\bar{\gamma}_{b} \ln (1-\delta)\right)$. Due to the fact that $p_{\mathrm{sop}}\left(R_{b}, R_{s}\right)$ is a decreasing function of $R_{b}$, we have $R_{b}=\log _{2}\left(1-\bar{\gamma}_{b} \ln (1-\delta)\right)$. From (26b) we also have $R_{b}-$ $\log _{2}\left(1-\bar{\gamma}_{e} \ln \eta\right) \geq R_{s}$, and therefore PF1 can be rewritten as

$$
\begin{aligned}
\text { PF1 : } \max _{R_{s}, \alpha, \beta} & p_{b}(\alpha, \beta) R_{s} \\
\text { s.t. } & R_{b}=\log _{2}\left(1-\bar{\gamma}_{b} \ln (1-\delta)\right) \\
& \mathbb{E}\left\{\left(1-I_{k}\right) C_{a}\right\}=\mathbb{E}\left\{I_{k} R_{s}\right\} \\
& R_{b}-\log _{2}\left(1-\bar{\gamma}_{e} \ln \eta\right) \geq R_{s}>0 \\
& \alpha \geq 0, \beta \geq 2^{R_{s}}-1,
\end{aligned}
$$

where by letting $R_{b}-\log _{2}\left(1-\bar{\gamma}_{e} \ln \eta\right) \geq 0$, a feasible solution exists if

$$
\delta \geq 1-\eta^{\frac{\overline{\bar{\gamma}_{e}}}{\bar{\gamma}_{b}}}
$$

Similarly, Problem PF2 with the extra parameter $R_{b}$ and an ROP constraint becomes

$$
\begin{aligned}
\text { PF2 }: \min _{R_{b}, R_{s}, \alpha, \beta} & p_{\text {sop }}\left(R_{b}, R_{s}\right) \\
\text { s.t. } & p_{b}(\alpha, \beta) R_{s} \geq \mu \\
& p_{\text {rop }}\left(R_{b}\right) \leq \delta \\
& \mathbb{E}\left\{\left(1-I_{k}\right) C_{a}\right\}=\mathbb{E}\left\{I_{k} R_{s}\right\} \\
& R_{b} \geq R_{s}>0, \alpha \geq 0, \beta \geq 2^{R_{s}}-1 .
\end{aligned}
$$

From (30c) and due to the fact that $p_{\mathrm{sop}}\left(R_{b}, R_{s}\right)$ is a decreasing function of $R_{b}$, we still have $R_{b}=\log _{2}\left(1-\bar{\gamma}_{b} \ln (1-\delta)\right)$, which allows us to reformulate PF2 as

$$
\begin{aligned}
& \text { PF2 : } \min _{R_{s}, \alpha, \beta} p_{\mathrm{sop}}\left(R_{b}, R_{s}\right) \\
& \text { s.t. } p_{b}(\alpha, \beta) R_{s} \geq \mu \\
& \mathbb{E}\left\{\left(1-I_{k}\right) C_{a}\right\}=\mathbb{E}\left\{I_{k} R_{s}\right\} \\
& R_{b}=\log _{2}\left(1-\bar{\gamma}_{b} \ln (1-\delta)\right) \\
& \\
& R_{b} \geq R_{s}>0, \alpha \geq 0, \beta \geq 2^{R_{s}}-1 .
\end{aligned}
$$

Both PF1 and PF2 can be solved via a two dimensional search over $\left\{R_{s}, \beta\right\}$, and given $R_{s}$ and $\beta$, the value of $\alpha$ can be obtained using (30d) or (31c). Note that the LS-TC scheme simply replaces $\beta$ with $2^{R_{s}}-1$ in (31), so we skip the discussion of LS-TC here and restrict our attention to LS-SC.

According to (19), (25), and (28), PF1 with LS-SC is given by

$$
\begin{aligned}
\mathbf{P F 1}(\mathrm{LS}-\mathrm{SC}): & \max _{R_{s}} \frac{R_{s}}{1+\lambda R_{s}} \\
\text { s.t. } R_{b}=\log _{2}\left(1-\bar{\gamma}_{b} \ln (1-\delta)\right) & \quad R_{b}-\log _{2}\left(1-\bar{\gamma}_{e} \ln \eta\right) \geq R_{s}>0 \\
& \ln \left(1+\lambda R_{s}\right) \geq \frac{\left(2^{R_{s}}-1\right)}{\bar{\gamma}_{b}},
\end{aligned}
$$

where $\lambda=\ln 2 / e^{1 / \bar{\gamma}_{a}} E_{1}\left(1 / \bar{\gamma}_{a}\right)$. This problem can be solved by using a line search over $R_{s}$. Furthermore, since (32a) is an increasing function of $R_{s}$, a closed-form solution $R_{s}^{*}=$ $\log _{2}\left(\left(1-\bar{\gamma}_{b} \ln (1-\delta)\right) /\left(1-\bar{\gamma}_{e} \ln \eta\right)\right)$ is valid if $R_{s}^{*}$ satisfies (32d).

According to (20), (25), and (31), Problem PF2 is given by

$$
\begin{array}{r}
\text { PF2(LS-SC) }: \min _{R_{b} \geq R_{s}>0} p_{\text {sop }}\left(R_{b}, R_{s}\right) \\
\text { s.t. } R_{s} \geq \mu /(1-\mu \lambda) \\
\quad R_{b}=\log _{2}\left(1-\bar{\gamma}_{b} \ln (1-\delta)\right) \\
\ln \left(1+\lambda R_{s}\right) \geq \frac{\left(2^{R_{s}}-1\right)}{\bar{\gamma}_{b}},
\end{array}
$$

and since $p_{\text {sop }}\left(R_{b}, R_{s}\right)$ increases as $R_{s}$ grows, the solution is expressed as $R_{s}^{*}=\mu /(1-\mu \lambda)$ if $R_{s}^{*}$ satisfies (33d). Otherwise a line search over $R_{s}$ is needed, and it can be observed that a feasible solution is conditioned on

$$
\delta \geq 1-e^{-\frac{2^{\frac{\mu}{1-\mu \lambda}}-1}{\bar{\gamma}_{b}}} .
$$

\section{B. Link Selection Without Reliability Outage}

Letting Bob send feedback when $C_{b}<R_{b}$, LS-OPT in (2) can be modified as

$$
I_{k \in\{1, \ldots, N\}}= \begin{cases}1 & \gamma_{b, k} \geq \max \left\{\alpha \gamma_{a, k}, \beta, 2^{R_{b}}-1\right\} \\ 0 & \text { otherwise. }\end{cases}
$$

Since $\beta$ and $R_{b}$ are constant parameters, the optimization can be divided into two cases, $\beta \geq 2^{R_{b}}-1$ and $\beta<2^{R_{b}}-1$, and the final result is then obtained by comparing these two cases.

When $\beta \geq 2^{R_{b}}-1$, the transmission probability $p_{b}$, the average arrival rate $\mathcal{E}\left\{\left(1-I_{k}\right) C_{a}\right\}$ and the SOP expressions are the same as in (3), (9) and (25) respectively. PF1 in (26) is then modified to

$$
\begin{aligned}
\text { PF1 }: \max _{R_{b}, R_{s}, \alpha, \beta} & p_{b}(\alpha, \beta) R_{s} \\
\text { s.t. } & p_{\text {sop }}\left(R_{b}, R_{s}\right) \leq \eta \\
& \mathbb{E}\left\{\left(1-I_{k}\right) C_{a}\right\}=\mathbb{E}\left\{I_{k} R_{s}\right\} \\
& R_{b} \geq R_{s}>0 \\
& \alpha \geq 0, \beta \geq 2^{R_{b}}-1 .
\end{aligned}
$$


Since (36e) implies $R_{b} \leq \log _{2}(1+\beta)$ and due to the fact that $p_{\text {sop }}\left(R_{b}, R_{s}\right)$ is a decreasing function of $R_{b}$, we have $R_{b}=$ $\log _{2}(1+\beta)$. From (36b) we also have $R_{b}-\log _{2}\left(1-\bar{\gamma}_{e} \ln \eta\right) \geq$ $R_{s}$, and therefore PF1 can be rewritten as

$$
\begin{aligned}
\text { PF1 : } \max _{R_{s}, \alpha, \beta} & p_{b}(\alpha, \beta) R_{s} \\
\text { s.t. } & R_{b}=\log _{2}(1+\beta) \\
& \mathbb{E}\left\{\left(1-I_{k}\right) C_{a}\right\}=\mathbb{E}\left\{I_{k} R_{s}\right\} \\
& R_{b}-\log _{2}\left(1-\bar{\gamma}_{e} \ln \eta\right) \geq R_{s}>0 \\
& \alpha \geq 0, \beta \geq 2^{R_{b}}-1 .
\end{aligned}
$$

Similarly, PF2 changes from (31) to

$$
\begin{aligned}
\text { PF2: } \min _{R_{s}, \alpha, \beta} & p_{\text {sop }}\left(R_{b}, R_{s}\right) \\
\text { s.t. } & p_{b}(\alpha, \beta) R_{s} \geq \mu \\
& \mathbb{E}\left\{\left(1-I_{k}\right) C_{a}\right\}=\mathbb{E}\left\{I_{k} R_{s}\right\} \\
& R_{b}=\log _{2}(1+\beta) \\
& R_{b} \geq R_{s}>0, \alpha \geq 0, \beta \geq 2^{R_{b}}-1 .
\end{aligned}
$$

When $\beta<2^{R_{b}}-1$, the transmission probability $p_{b}$, and the average arrival rate $\mathcal{E}\left\{\left(1-I_{k}\right) C_{a}\right\}$ are simply modifications of (3) and (9) with $\beta$ replaced by $2^{R_{b}}-1$. The SOP expression remains the same as (25). Therefore, $p_{b}\left(\alpha, R_{b}\right), p_{\text {sop }}\left(R_{b}, R_{s}\right)$ and $\mathcal{E}\left\{\left(1-I_{k}\right) C_{a}\right\}$ are not functions of $\beta$ and PF1 and PF2 become

$$
\begin{aligned}
\text { PF1: } \max _{R_{s}, \alpha, R_{b}} & p_{b}\left(\alpha, R_{b}\right) R_{s} \\
\text { s.t. } & \mathbb{E}\left\{\left(1-I_{k}\right) C_{a}\right\}=\mathbb{E}\left\{I_{k} R_{s}\right\} \\
& R_{b}-\log _{2}\left(1-\bar{\gamma}_{e} \ln \eta\right) \geq R_{s}>0 \\
& \alpha \geq 0
\end{aligned}
$$

and

$$
\begin{aligned}
& \text { PF2 : } \min _{R_{s}, \alpha, R_{b}} p_{\text {sop }}\left(R_{b}, R_{s}\right) \\
& \text { s.t. } p_{b}\left(\alpha, R_{b}\right) R_{s} \geq \mu \\
& \mathbb{E}\left\{\left(1-I_{k}\right) C_{a}\right\}=\mathbb{E}\left\{I_{k} R_{s}\right\} \\
& R_{b} \geq R_{s}>0, \alpha \geq 0 .
\end{aligned}
$$

As in Section V-A, for the case of $\beta \geq 2^{R_{b}}-1$, both PF1 and PF2 above can be solved via a two dimensional search over $\left\{R_{s}, \beta\right\}$, and given $R_{s}$ and $\beta$, the value of $\alpha$ can be obtained using $\mathbb{E}\left\{\left(1-I_{k}\right) C_{a}\right\}=\mathbb{E}\left\{I_{k} R_{s}\right\}$. For case of $\beta<2^{R_{b}}-1$, PF1 and PF2 are solved via a two dimensional search over $\left\{R_{s}, R_{b}\right\}$, and given $R_{s}$ and $R_{b}$, the value of $\alpha$ can be obtained using $\mathbb{E}\left\{\left(1-I_{k}\right) C_{a}\right\}=\mathbb{E}\left\{I_{k} R_{s}\right\}$.

\section{BufFered Relaying With Delay Constraints}

Thus far, we have assumed that the buffer at the relay is of essentially unlimited length, and we have ignored the impact of buffering on the transmission delay between Alice and Bob. A practical implementation however would employ a finitelength buffer and may impose constraints on the maximum delay that can be tolerated. If the buffer is near overflow or the communication delay is near the maximum allowable value, the relay may be forced to transmit regardless of the channel conditions. The development of an optimal policy that takes these factors into account, and a precise analysis of the resulting delay vs. secrecy trade-off, are challenging and beyond the scope of this paper. Instead, here we will analyze the impact of a relatively simple method for taking delay constraints or a limited buffer size into account.

Let $Q_{\max }$ represent the fixed (finite) length of the buffer, and consider for example the following modification of the selection criterion for LS-OPT given in (2):

$$
I_{k}^{Q_{\max }}= \begin{cases}1 & \gamma_{b, k} \geq \max \left\{\alpha \gamma_{a, k}, \beta\right\} \\ & \text { or }\left(Q_{\max }-Q_{k-1}\right)<R_{a, k} \\ 0 & \text { otherwise }\end{cases}
$$

where $\left(Q_{\max }-Q_{k-1}\right)$ represents the available room in the buffer measured in bits, and $R_{a, k}$ is the size in bits of the incoming packet at time slot $k$ if Alice is chosen to transmit. A scheme similar to (41) could also be implemented for the LS-TC and LS-SC methods as well. Using Little's Law [35], the average delay of this buffered relaying approach is given by

$$
\bar{L}=\frac{\mathbb{E}\left[Q_{k}\right]}{R_{\text {in }}},
$$

where $\mathbb{E}\left[Q_{k}\right]$ is the average queue length in bits and $R_{\text {in }}$ is the average arrival rate defined in (4) in bits/slot, so the average delay $\bar{L}$ is in slots. The approach in (41) could be further modified to force transmission if the delay has exceeded a particular threshold. In the next section, we provide some simulation examples to illustrate the performance of this link selection approach.

\section{NumericAl Results AND Discussions}

In this section, we present numerical examples of the security performance for the proposed link selection schemes, LS-OPT, LS-TC, and LS-SC in both adaptive-rate and fixed-rate relaying scenarios. The secrecy throughput and the SOP of these schemes are demonstrated for various values of the secrecy constraints, desired throughput, and average SNRs.

\section{A. Conventional Unbuffered Relaying}

In order to show the security improvement introduced by buffer-aided relaying, we compare its performance with the conventional unbuffered approach. In conventional two-hop relaying, Alice transmits data in one time slot and the relay decodes the data, reencodes it with a secrecy codebook, and forwards it to Bob in the next time slot. The secrecy throughput is given by

$$
R_{t}=\lim _{N \rightarrow \infty} \frac{1}{N} \sum_{k=1}^{N / 2} R_{2 k}
$$

where for the $2 k$-th time slot,

$$
R_{2 k}= \begin{cases}\min \left\{C_{a, 2 k-1}, R_{s}\right\} & \text { if } \min \left\{C_{a, 2 k-1}, R_{s}\right\}<C_{b, 2 k} \\ 0 & \text { otherwise }\end{cases}
$$




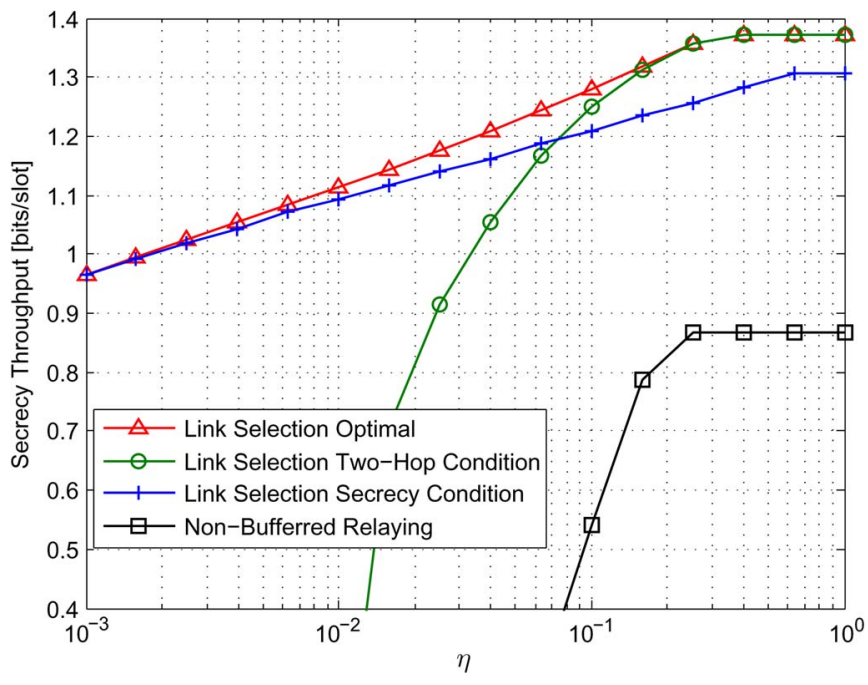

Fig. 2. Secrecy throughput versus desired SOP constraint $\eta$, adaptiverate transmission at the relay, Problem P1 with $\bar{\gamma}_{a}=5 \mathrm{~dB}, \bar{\gamma}_{b}=15 \mathrm{~dB}$, $\bar{\gamma}_{e}=0 \mathrm{~dB}$.

due to the fact that when $\min \left\{C_{a, 2 k-1}, R_{s}\right\} \geq C_{b, 2 k}$, there is no feasible secrecy codebook at the relay and the data packets must be dropped. It is easily seen that the secrecy throughput is upper bounded by $(1 / 2) R_{s}$. Also, the SOP in this case is lower bounded by

$$
p_{\text {sop }}\left(R_{s}\right) \geq \operatorname{Pr}\left[C_{e}>C_{b}-\min \left\{C_{a}, R_{s}\right\}\right] .
$$

This is a lower bound since unlike buffered relaying that can flexibly choose good time slots for transmission, the unbuffered case cannot guarantee $C_{b}>\min \left\{C_{a}, R_{s}\right\}$ during the relay transmission phase. Therefore, when $C_{b} \leq \min \left\{C_{a}, R_{s}\right\}$, the relay cannot transmit any private data and it cannot be considered as a secrecy outage event even if $C_{e}>C_{b}-$ $\min \left\{C_{a}, R_{s}\right\}$. For simplicity, in the simulations we will only evaluate the above bounds on secrecy throughput and SOP for unbuffered relaying rather than computing their actual values, and we will see that the performance gain of buffered relaying is still obvious even when compared with the bounds for the unbuffered case.

\section{B. Adaptive-Rate Buffered Relaying}

Fig. 2 shows the secrecy throughput versus the desired SOP constraint (Problem P1) for various link selection polices, assuming average SNRs of $\bar{\gamma}_{a}=5 \mathrm{~dB}, \bar{\gamma}_{b}=15 \mathrm{~dB}, \bar{\gamma}_{e}=0 \mathrm{~dB}$, and assuming an adaptive rate at the relay. As expected in general for all schemes, a higher $\eta$ results in a larger secrecy throughput. When $\eta$ is close to 1 , the performance of all schemes saturates to the conventional throughput without secrecy constraints. We can also see that the throughput gain of buffered over unbuffered relaying is significant. The performance of LS-OPT is always the best among all schemes, and it is observed that the performance of LS-SC converges to that of LS-OPT when $\eta$ is low, while LS-TC converges to LS-OPT as $\eta$ grows. This indicates that the selection parameter $\beta$ dominates with a stricter secrecy constraint, and $\alpha$ dominates in turn when the secrecy constraint is more relaxed.

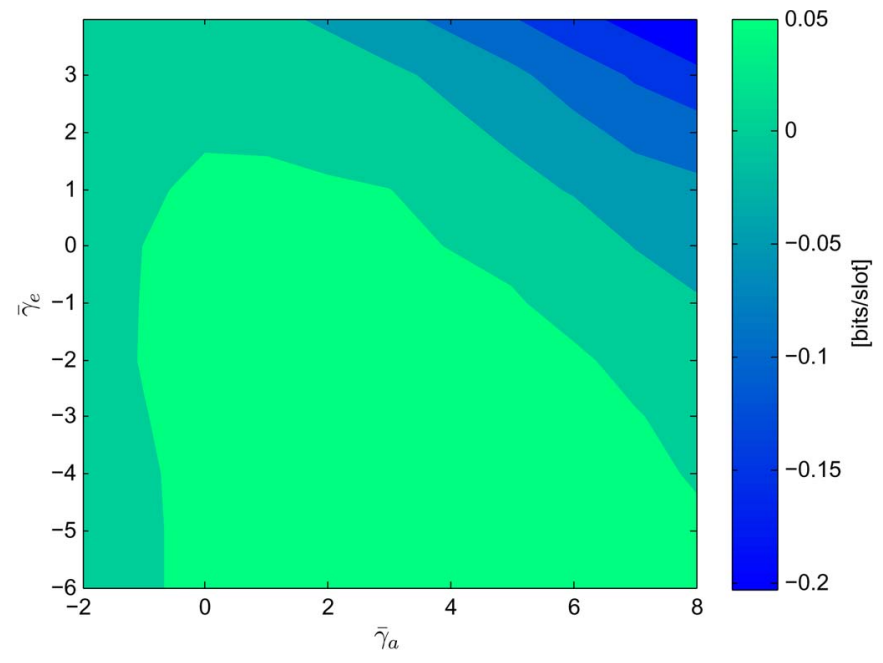

Fig. 3. Secrecy throughput difference between LS-TC and LS-SC versus average SNRs $\bar{\gamma}_{a}$ and $\bar{\gamma}_{e}$, adaptive-rate transmission at the relay, Problem P1 with $\bar{\gamma}_{b}=15 \mathrm{~dB}, \eta=0.1$.

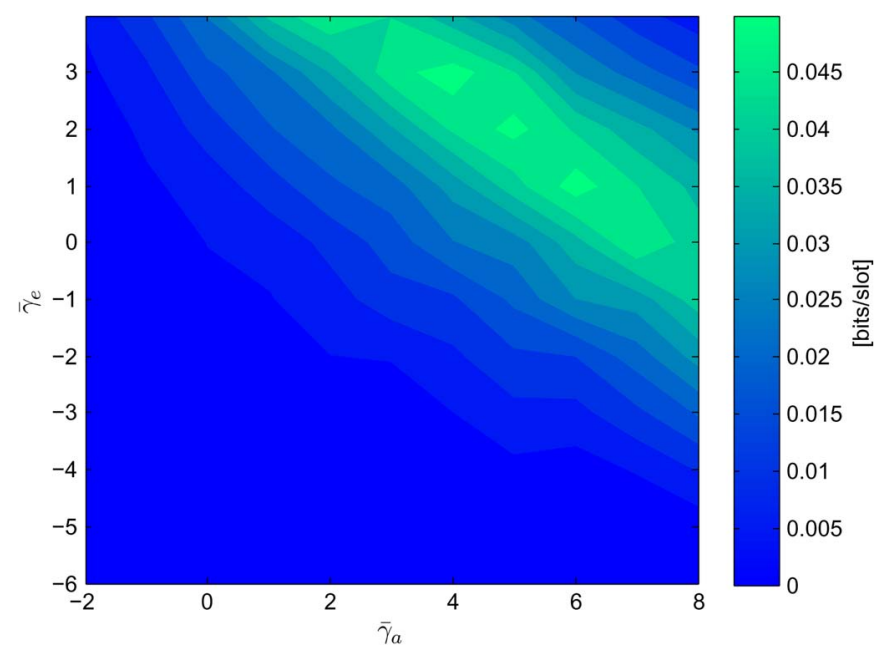

Fig. 4. Secrecy throughput difference between LS-OPT and $\max \{$ LS-TC, LSSC $\}$ versus average SNRs $\bar{\gamma}_{a}$ and $\bar{\gamma}_{e}$, adaptive-rate transmission at the relay, Problem P1 with $\bar{\gamma}_{b}=15 \mathrm{~dB}, \eta=0.1$.

The variations in secrecy throughput for different link selection schemes are depicted in Figs. 3 and 4, for various values of average SNR in the Alice-Relay and Relay-Eve links. Fig. 3 compares the throughput of LS-TC and LS-SC when $\bar{\gamma}_{b}=15 \mathrm{~dB}$ and $\eta=0.1$. It can be seen that for a fixed value of $\bar{\gamma}_{e}$, a higher $\bar{\gamma}_{a}$ results in better performance for LS-SC. This is because when $\bar{\gamma}_{a}$ is closer to $\bar{\gamma}_{b}(15 \mathrm{~dB}$ in this case), the two-hop conditions are more balanced and the design of $\alpha$ becomes less important compared to $\beta$. Also observe that a higher $\bar{\gamma}_{e}$ corresponds to better performance for LS-SC since the design of $\beta$ is more important when the eavesdropper's wiretapping ability becomes stronger. Fig. 4 shows the throughput difference between LS-OPT and the superior of the two sub-optimal schemes. We see that the optimal scheme only outperforms the suboptimal ones over a relatively moderate range of values for $\bar{\gamma}_{a}$ and $\bar{\gamma}_{e}$. When $\bar{\gamma}_{a / e}$ becomes lower or higher, the performance of LS-TC and LS-SC will respectively approach that of LS-OPT. 


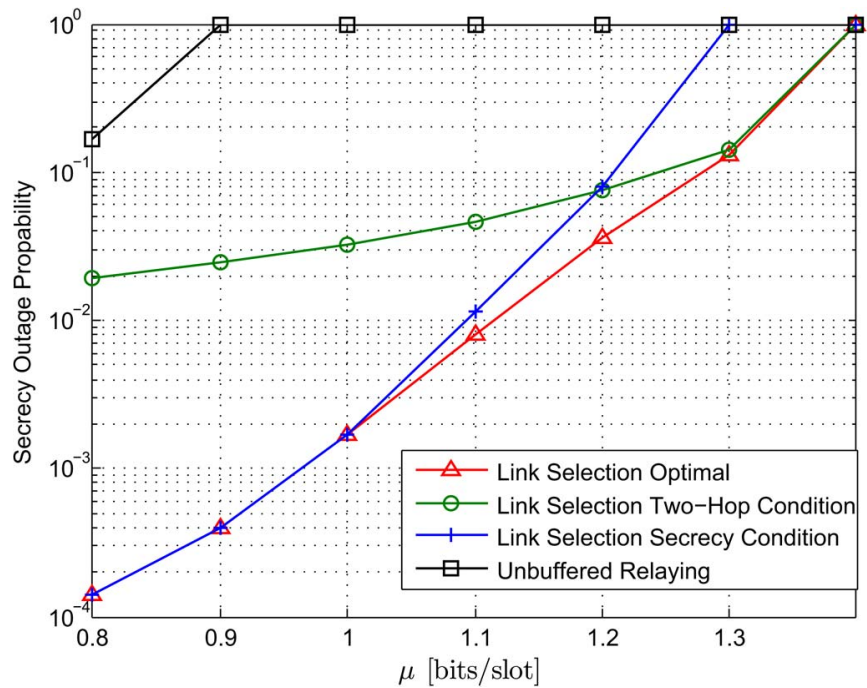

Fig. 5. Secrecy outage probability versus desired secrecy throughput constraint $\mu$, adaptive-rate transmission at the relay, Problem P2 with $\bar{\gamma}_{a}=5 \mathrm{~dB}$, $\bar{\gamma}_{b}=15 \mathrm{~dB}, \bar{\gamma}_{e}=0 \mathrm{~dB}$.

Fig. 5 illustrates the SOP as a function of the desired secrecy throughput threshold $\mu$ (Problem P2) assuming $\bar{\gamma}_{a}=5 \mathrm{~dB}$, $\bar{\gamma}_{b}=15 \mathrm{~dB}, \bar{\gamma}_{e}=0 \mathrm{~dB}$. It shows a significant performance gain for buffered relaying compared with the unbuffered case. For example, when $\mu \geq 0.9 \mathrm{bps} / \mathrm{Hz}$, unbuffered relaying hardly allows any secure transmission while LS-OPT can still guarantee an SOP as low as $10^{-3}$. Recall that the SOP for unbuffered relaying plotted here is just a lower bound, so the actual gain could be even larger. When $\mu$ is small, the SOP of LS-TC is obviously worse than that of other selection schemes, and the SOP of LS-SC converges to that of LS-OPT. On the other hand, when we require a larger $\mu$, the performance of LS-SC degrades and LS-TC converges to LS-OPT, since it seeks a better throughput.

\section{Fixed-Rate Buffered Relaying}

Fig. 6 shows the secrecy throughput of the link selection schemes for the case of fixed-rate transmission at the relay, assuming $\bar{\gamma}_{a}=20 \mathrm{~dB}, \bar{\gamma}_{b}=10 \mathrm{~dB}, \bar{\gamma}_{e}=0 \mathrm{~dB}$, and the ROP constraint $\delta=0.1$. Compared to Fig. 2, it is seen that the overall secrecy throughput is lower, and the performance gain of buffered vs. unbuffered relaying is also smaller. Interestingly, we observe that the performance of LS-OPT and LS-TC is the same and is superior to LS-SC for the entire range of $\eta$, which indicates that the optimal $\beta$ for LS-OPT is always $2^{R_{s}}-1$. This can also be inferred from (26), where $p_{\text {sop }}$ is not a function of $\beta$ (unlike the adaptive-rate case), so $\beta$ should always be set to its lower bound $2^{R_{s}}-1$ in order to maximize the transmission probability, as long as (26d) is satisfied. We can also see the same result in Fig. 7, where LS-OPT and LS-TC demonstrate identically low secrecy outage performance. Therefore, for the fixed-rate scenario, one should adopt LS-TC for reduced complexity.

\section{Buffered Relaying With Delay Constraints}

In Fig. 8, we show the secrecy throughput as a function of average delay by varying the buffer size at the relay. For a smaller

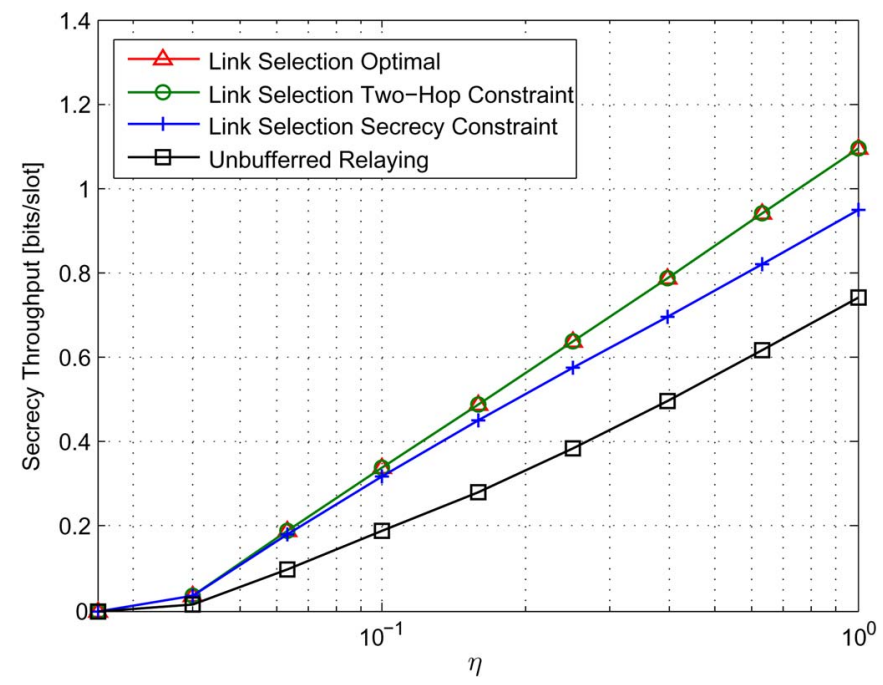

Fig. 6. Secrecy throughput versus desired SOP constraint $\eta$, fixed-rate transmission at the relay, Problem PF1 with $\bar{\gamma}_{a}=5 \mathrm{~dB}, \bar{\gamma}_{b}=15 \mathrm{~dB}, \bar{\gamma}_{e}=0 \mathrm{~dB}$, ROP constraint $\delta=0.1$.

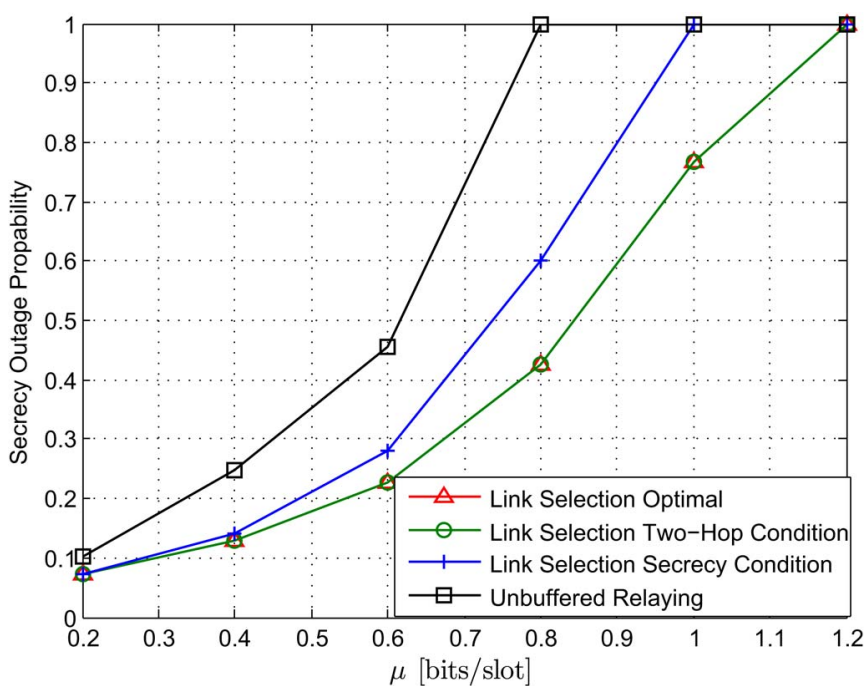

Fig. 7. Secrecy outage probability versus desired secrecy throughput constraint $\mu$, fixed-rate transmission at the relay, Problem PF2 with $\bar{\gamma}_{a}=5 \mathrm{~dB}$, $\bar{\gamma}_{b}=15 \mathrm{~dB}, \bar{\gamma}_{e}=0 \mathrm{~dB}$, ROP constraint $\delta=0.1$.

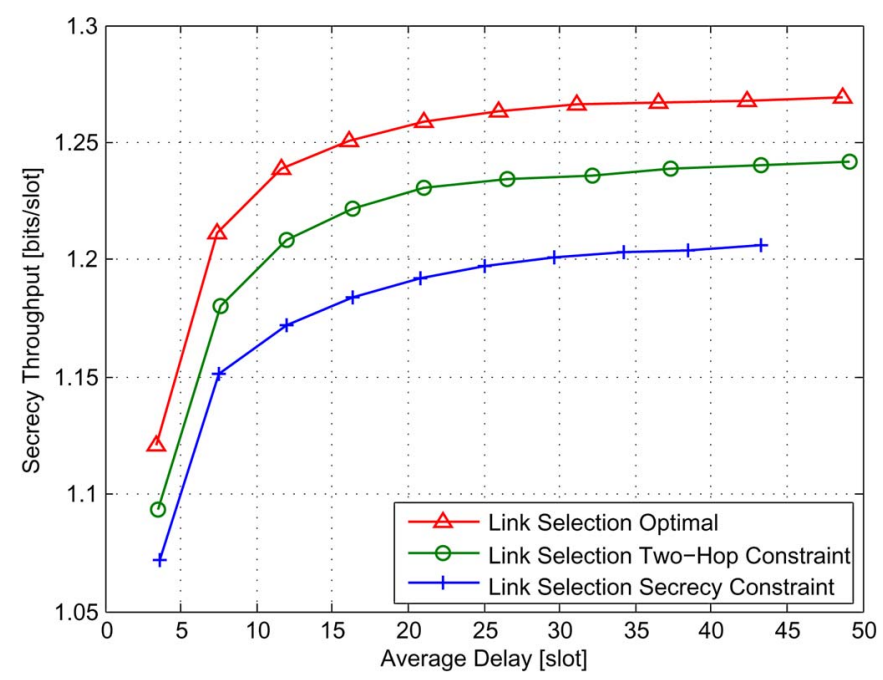

Fig. 8. Secrecy throughput versus average delay of buffered relaying, with $\bar{\gamma}_{a}=5 \mathrm{~dB}, \bar{\gamma}_{b}=15 \mathrm{~dB}, \bar{\gamma}_{e}=0 \mathrm{~dB}$, and SOP constraint $\eta=0.1$. 
delay constraint, we observe that secure relaying is relatively inefficient in terms of secrecy throughput because with a shorter buffer size, the relay is more likely to transmit according to the selection criterion in (41). On the other hand, when the delay constraint is more relaxed, the secrecy throughput increases and gradually approaches the result in Fig. 2 with an unlimited buffer. In this case, it appears that a buffer designed to handle the data from 25 or more time slots has essentially the same secrecy performance as one of infinite length. The example shows that LS-OPT has the highest secrecy throughput; with $\eta=0.1$ as in this example, LS-TC is more efficient than LS-SC, but it is expected that when $\eta<0.05$, LS-SC will give higher secrecy throughput than LS-TC, as inferred from Fig. 2.

\section{CONCLUSION}

This paper has considered a two-hop DF relay network where the relay can be wiretapped by an eavesdropper. We assume that the eavesdropper's instantaneous CSI is not available at the relay, and the relay is aided by a buffer and thus can dynamically control whether each time slot is used for reception or transmission. We proposed a link selection policy that takes into account both the two-hop rate balance and the security constraint, and we optimized the link selection parameters based on the closed-form expressions derived for the secrecy throughput and SOP. We also discussed two suboptimal link selection schemes based on the optimal approach that only consider either the two-hop conditions (LS-TC) or the secrecy constraint (LS-SC). We show that the performance of the reduced complexity LS-TC method approaches optimality with a more relaxed secrecy constraint while LS-SC reaches optimal performance with a stricter secrecy constraint. We also showed that LS-TC always provides optimal performance in the scenario where the relay does not know Bob's CSI and thus uses a fixed-rate transmission scheme. We further presented a simple approach for modifying the proposed methods when delay constraints are present or the size of the buffer is limited. Various numerical examples were used to demonstrate that buffer-aided relaying provides flexibility that can lead to significant performance improvement in terms of both secrecy throughput and secrecy outage probability.

\section{APPENDIX A}

\section{PROOF OF PROPOSITION 1}

Denoting the p.d.f.s of $\gamma_{a}$ and $\gamma_{b}$ as $f_{a}\left(\gamma_{a}\right)$ and $f_{b}\left(\gamma_{a}\right)$, the average arrival rate at the relay is

$$
\begin{aligned}
\mathbb{E} & \left\{\left(1-I_{k}\right) R_{a}\right\} \\
= & \int_{0}^{\beta}\left(\int_{0}^{\infty} \log _{2}\left(1+\gamma_{a}\right) f_{a}\left(\gamma_{a}\right) d \gamma_{a}\right) f_{b}\left(\gamma_{b}\right) d \gamma_{b} \\
& +\int_{\beta}^{\infty}\left(\int_{\frac{\gamma_{b}}{\alpha}}^{\infty} \log _{2}\left(1+\gamma_{a}\right) f_{a}\left(\gamma_{a}\right) d \gamma_{a}\right) f_{b}\left(\gamma_{b}\right) d \gamma_{b} \\
= & \nu(\beta)+\phi(\alpha, \beta),
\end{aligned}
$$

where

$$
\begin{aligned}
\nu(\beta) & =\int_{0}^{\beta}\left(\int_{0}^{\infty} \log _{2}\left(1+\gamma_{a}\right) f_{a}\left(\gamma_{a}\right) d \gamma_{a}\right) f_{b}\left(\gamma_{b}\right) d \gamma_{b} \\
& =\left(1-e^{-\frac{\beta}{\bar{\gamma}_{b}}}\right) \int_{0}^{\infty} \frac{1}{\ln 2} \frac{1}{1+\gamma_{a}} e^{-\frac{\gamma_{a}}{\bar{\gamma}_{a}}} d \gamma_{a} \\
& =\frac{1}{\ln 2}\left(1-e^{-\frac{\beta}{\bar{\gamma}_{b}}}\right) e^{\frac{1}{\overline{\gamma_{a}}}} \int_{1}^{\infty} \frac{1}{x} e^{-\frac{x}{\bar{\gamma}_{a}}} d x \\
& =\frac{1}{\ln 2}\left(1-e^{-\frac{\beta}{\bar{\gamma}_{b}}}\right) e^{\frac{1}{\bar{\gamma}_{a}}} E_{1}\left(\frac{1}{\bar{\gamma}_{a}}\right),
\end{aligned}
$$

and

$$
\begin{aligned}
& \phi(\alpha, \beta) \\
& =\int_{\beta}^{\infty}\left(\int_{\frac{\gamma_{b}}{\alpha}}^{\infty} \log _{2}\left(1+\gamma_{a}\right) f_{a}\left(\gamma_{a}\right) d \gamma_{a}\right) f_{b}\left(\gamma_{b}\right) d \gamma_{b} \\
& \underline{\underline{a}} \int_{\beta}^{\infty} \frac{1}{\bar{\gamma}_{a}}\left[\left.\log _{2}\left(1+\gamma_{a}\right)\left(-\bar{\gamma}_{a} e^{-\frac{\gamma_{a}}{\bar{\gamma}_{a}}}\right)\right|_{\frac{\gamma_{b}}{\alpha}} ^{\infty}\right. \\
& \left.\quad-\int_{\frac{\gamma_{b}}{\alpha}}^{\infty} \frac{1}{\ln 2} \frac{1}{1+\gamma_{a}}\left(-\bar{\gamma}_{a} e^{-\frac{\gamma_{a}}{\bar{\gamma}_{a}}}\right) d \gamma_{a}\right] \frac{1}{\bar{\gamma}_{b}} e^{-\frac{\gamma_{b}}{\bar{\gamma}_{b}}} d \gamma_{b} \\
& \int_{\beta}^{\infty} \frac{1}{\bar{\gamma}_{b}} \log _{2}\left(1+\frac{\gamma_{b}}{\alpha}\right) e^{-\left(\frac{\gamma_{b}}{\alpha \bar{\gamma}_{a}}+\frac{\gamma_{b}}{\bar{\gamma}_{b}}\right)} d \gamma_{b} \\
& +\int_{\beta}^{\infty} \frac{1}{\ln 2} \frac{1}{\bar{\gamma}_{b}} e^{\frac{1}{\bar{\gamma}_{a}}} E_{1}\left(\frac{\gamma_{b}}{\alpha \bar{\gamma}_{a}}+\frac{1}{\bar{\gamma}_{a}}\right) e^{-\frac{\gamma_{b}}{\bar{\gamma}_{b}}} d \gamma_{b} \\
& =g_{1}(\alpha, \beta)+g_{2}(\alpha, \beta),
\end{aligned}
$$

where integration by parts is used in equality (a) and Eq. 3.352.2 in [36] is used to obtain equality (b). With further manipulations, $g_{1}(\alpha, \beta)$ can be calculated as

$$
\begin{aligned}
& g_{1}(\alpha, \beta) \\
& =\int_{\beta}^{\infty} \frac{1}{\bar{\gamma}_{b}} \log _{2}\left(1+\frac{\gamma_{b}}{\alpha}\right) e^{-\left(\frac{\gamma_{b}}{\alpha \bar{\gamma}_{a}}+\frac{\gamma_{b}}{\bar{\gamma}_{b}}\right)} d \gamma_{b} \\
& =\int_{\frac{\beta}{\alpha}}^{\infty} \frac{\alpha}{\bar{\gamma}_{b}} \log _{2}(1+x) e^{-\left(\frac{1}{\bar{\gamma}_{a}}+\frac{\alpha}{\bar{\gamma}_{b}}\right) x} d x \\
& =\log _{2}\left(1+\frac{\beta}{\alpha}\right) \frac{\overline{\alpha \gamma_{a}}}{\alpha \bar{\gamma}_{a}+\bar{\gamma}_{b}} e^{-\left(\frac{\beta}{\alpha \bar{\gamma}_{a}}+\frac{\beta}{\bar{\gamma}_{b}}\right)} \\
& \quad+\frac{1}{\ln 2} \frac{\alpha \bar{\gamma}_{a}}{\alpha \bar{\gamma}_{a}+\bar{\gamma}_{b}} e^{\left(\frac{1}{\bar{\gamma}_{a}}+\frac{\alpha}{\bar{\gamma}_{b}}\right)} E_{1}\left[\left(\frac{1}{\bar{\gamma}_{a}}+\frac{\alpha}{\bar{\gamma}_{b}}\right)\left(\frac{\beta}{\alpha}+1\right)\right]
\end{aligned}
$$


where the variable exchange $x=\gamma_{b} / \alpha$ is used and

$$
\begin{aligned}
g_{2}(\alpha, \beta)= & \int_{\beta}^{\infty} \frac{1}{\ln 2} \frac{1}{\bar{\gamma}_{b}} e^{\frac{1}{\bar{\gamma}_{a}}} E_{1}\left(\frac{\gamma_{b}}{\alpha \bar{\gamma}_{a}}+\frac{1}{\bar{\gamma}_{a}}\right) e^{-\frac{\gamma_{b}}{\bar{\gamma}_{b}}} d \gamma_{b} \\
= & \int_{\alpha+\beta}^{\infty} \frac{1}{\ln 2} \frac{1}{\bar{\gamma}_{b}} e^{\left(\frac{1}{\bar{\gamma}_{a}}+\frac{\alpha}{\bar{\gamma}_{b}}\right)} E_{1}\left(\frac{x}{\alpha \bar{\gamma}_{a}}\right) e^{-\frac{x}{\bar{\gamma}_{b}}} d x \\
= & \frac{1}{\ln 2} e^{\left(\frac{1}{\bar{\gamma}_{a}}+\frac{\alpha}{\bar{\gamma}_{b}}\right)}\left\{e^{-\frac{\alpha+\beta}{\bar{\gamma}_{b}}} E_{1}\left(\frac{\alpha+\beta}{\alpha \bar{\gamma}_{a}}\right)\right. \\
& \left.-E_{1}\left[\left(\frac{1}{\bar{\gamma}_{a}}+\frac{\alpha}{\bar{\gamma}_{b}}\right)\left(\frac{\beta}{\alpha}+1\right)\right]\right\}
\end{aligned}
$$

where $x=\alpha+\gamma_{b}$ and 4.2.1 in [37] are used to obtain the final equality. Combining the above equations, the results in this proposition are proved.

\section{REFERENCES}

[1] J. N. Laneman, D. N. C. Tse, and G. W. Wornell, "Cooperative diversity in wireless networks: Efficient protocols and outage behavior," IEEE Trans. Inf. Theory, vol. 50, no. 12, pp. 3062-3080, Dec. 2004.

[2] A. Host-Madsen and J. Zhang, "Capacity bounds and power allocation for wireless relay channels," IEEE Trans. Inf. Theory, vol. 51, no. 6, pp. 2020-2040, Jun. 2005.

[3] A. Mukherjee, S. Fakoorian, J. Huang, and A. L. Swindlehurst, "Principles of physical layer security in multiuser wireless networks: A survey," IEEE Commun. Surveys Tuts., to be published.

[4] L. Dong, Z. Han, A. P. Petropulu, and H. V. Poor, "Improving wireless physical layer security via cooperating relays," IEEE Trans. Signal Process., vol. 58, no. 3, pp. 1875-1888, Mar. 2010.

[5] Y. Yang, Q. Li, W.-K. Ma, J. Ge, and P. C. Ching, "Cooperative secure beamforming for AF relay networks with multiple eavesdroppers," IEEE Signal Process. Lett., vol. 20, no. 1, pp. 35-38, Jan. 2013.

[6] S. Goel and R. Negi, "Guaranteeing secrecy using artificial noise," IEEE Trans. Wireless Commun., vol. 7, no. 6, pp. 2180-2189, Jun. 2008.

[7] G. Zheng, L.-C. Choo, and K.-K. Wong, "Optimal cooperative jamming to enhance physical layer security using relays," IEEE Trans. Signal Process., vol. 59, no. 3, pp. 1317-1322, Mar. 2011.

[8] J. Zhang and M. C. Gursoy, "Collaborative relay beamforming for secrecy," in Proc. IEEE ICC, May 2010, pp. 1-5.

[9] I. Krikidis, J. Thompson, and S. Mclaughlin, "Relay selection for secure cooperative networks with jamming," IEEE Trans. Wireless Commun., vol. 8, no. 10, pp. 5003-5011, Oct. 2009.

[10] Z. Ding, K. K. Leung, D. L. Goeckel, and D. Towsley, "Opportunistic relaying for secrecy communications: Cooperative jamming vs. relay chatting," IEEE Trans. Wireless Commun., vol. 10, no. 6, pp. 1725-1729, Jun. 2011.

[11] J. Huang and A. L. Swindlehurst, "Cooperative jamming for secure communications in MIMO relay networks," IEEE Trans. Signal Process., vol. 59, no. 10, pp. 4871-4884, Oct. 2011.

[12] H.-M. Wang, M. Luo, X.-G. Xia, and Q. Yin, "Joint cooperative beamforming and jamming to secure AF relay systems with individual power constraint and no eavesdropper's CSI," IEEE Signal Process. Lett., vol. 20, no. 1, pp. 39-42, Jan. 2013.

[13] Z. Ding, Z. Ma, and P. Fan, "Asymptotic studies for the impact of antenna selection on secure two-way relaying communications with artificial noise," IEEE Trans. Wireless Commun., vol. 13, no. 4, pp. 2189-2203, Apr. 2014

[14] H.-M. Wang, Q. Yin, and X.-G. Xia, "Distributed beamforming for physical-layer security of two-way relay networks," IEEE Trans. Signal Process., vol. 60, no. 7, pp. 3532-3545, Jul. 2012.

[15] X. He and A. Yener, "Cooperation with an untrusted relay: A secrecy perspective," IEEE Trans. Inf. Theory, vol. 56, no. 8, pp. 3807-3827, Aug. 2010.

[16] C. Jeong, I.-M. Kim, and D. I. Kim, "Joint secure beamforming design at the source and the relay for an amplify-and-forward MIMO untrusted relay system," IEEE Trans. Signal Process., vol. 60, no. 1, pp. 310-325, Jan. 2012.
[17] J. Huang, A. Mukherjee, and A. L. Swindlehurst, "Secure communication via an untrusted non-regenerative relay in fading channels," IEEE Trans. Signal Process., vol. 61, no. 10, pp. 2536-2550, May 2013.

[18] A. Mukherjee, "Imbalanced beamforming by a multi-antenna source for secure utilization of an untrusted relay," IEEE Commun. Lett., vol. 17, no. 7, pp. 1309-1312, Jul. 2013.

[19] B. Xia, Y. Fan, J. Thompson, and H. V. Poor, "Buffering in a three-node relay network," IEEE Trans. Wireless Commun., vol. 7, no. 11, pp. 4492 4496, Nov. 2008.

[20] A. Ikhlef, D. S. Michalopoulos, and R. Schober, "Max-max relay selection for relays with buffers," IEEE Trans. Wireless Commun., vol. 11, no. 3, pp. 1124-1135, Mar. 2012.

[21] N. Zlatanov, R. Schober, and P. Popovski, "Throughput and diversity gain of buffer-aided relaying," in Proc. GLOBECOM, Dec. 2011, pp. 1-6.

[22] I. Krikidis, T. Charalambous, and J. S. Thompson, "Buffer-aided relay selection for cooperative diversity systems without delay constraints," IEEE Trans. Wireless Commun., vol. 11, no. 5, pp. 1957-1967, May 2012.

[23] N. Zlatanov, R. Schober, and P. Popovski, "Buffer-aided relaying with adaptive link selection," IEEE J. Sel. Areas Commun., vol. 59, no. 5, pp. 2816-2840, May 2013.

[24] P. K. Gopala, L. Lai, and H. El Gamal, "On the secrecy capacity of fading channels," IEEE Trans. Inf. Theory, vol. 54, no. 10, pp. 4687-4698, Oct. 2008.

[25] X. Zhou, M. R. McKay, B. Maham, and A. Hjørungnes, "Rethinking the secrecy outage formulation: A secure transmission design perspective," IEEE Commun. Lett., vol. 15, no. 3, pp. 302-304, Mar. 2011.

[26] G. Chen, Z. Tian, Y. Gong, Z. Chen, and J. A. Chambers, "Max-ratio relay selection in secure buffer-aided cooperative wireless networks," IEEE Trans. Inf. Forensics Security, vol. 9, no. 4, pp. 719-729, Apr. 2014.

[27] L. Dong, Z. Han, A. P. Petropulu, and H. V. Poor, "Secure wireless communications via cooperation," in Proc. 46th Annu. Allerton Conf. Commun., Control, Comput., 2008, pp. 1132-1138.

[28] M. Bloch, J. Barros, M. Rodrigues, and S. McLaughlin, "Wireless information-theoretic security," IEEE Trans. Inf. Theory, vol. 54, no. 6, pp. 2515-2534, Jun. 2008.

[29] X. Zhang, X. Zhou, and M. R. McKay, "On the design of artificial-noiseaided secure multi-antenna transmission in slow fading channels," IEEE Trans. Veh. Technol., vol. 62, no. 5, pp. 2170-2181, Jun. 2013.

[30] Y. Sarikaya and O. Ercetin, "On physically secure and stable slotted aloha system," in Proc. 47th Annu. Allerton Conf. Commun., Control, Comput., Sep. 2009, pp. 1090-1096.

[31] Y. Liang, H. V. Poor, and L. Ying, "Secure communications over wireless broadcast networks: Stability and utility maximization," IEEE Trans. Inf. Forensics Security, vol. 6, no. 3, pp. 682-692, Sep. 2011.

[32] M. Ozmen and M. C. Gursoy, "Throughput and energy efficiency under queueing and secrecy constraints," in Proc. 46th Asilomar Conf. Signals, Syst. Comput., Nov. 2012, pp. 63-67.

[33] Z. Mao, C. E. Koksal, and N. B. Shroff, "Achieving full secrecy rate with low packet delays: An optimal control approach," IEEE J. Sel. Areas Commun., vol. 31, no. 9, pp. 1944-1956, Sep. 2013.

[34] X. Tang, R. Liu, P. Spasojevic, and H. V. Poor, "On the throughput of secure hybrid-ARQ protocols for Gaussian block-fading channels," IEEE Trans. Inf. Theory, vol. 55, no. 4, pp. 1575-1591, Apr. 2009.

[35] L. Kleinrock, Queueing Systems Volume 1: Theory. Hoboken, NJ, USA: Wiley, 1975 .

[36] I. S. Gradshteyn and I. M. Ryzhik, Tables of Integrals, Series, Products., 7th ed. San Diego, CA, USA: Academic, 2007.

[37] M. Geller and E. W. Ng, "A table of integrals of exponential integral," $J$. Res. Nat. Bureau Std., vol. 73B, no. 3, pp. 191-210, Sep. 1969.

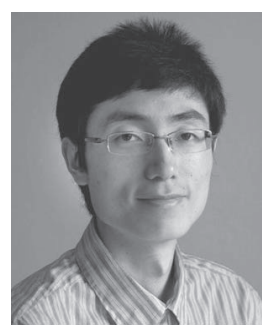

Jing Huang (S'10-M'13) received the B.S. degree in communication engineering from Jilin University, Changchun, China, in 2006; the M.S. degree in communications and information systems from Beijing University of Posts and Telecommunications, Beijing, China, in 2009; and the Ph.D. degree in electrical and computer engineering from the University of California, Irvine, CA, in 2013.

During Spring and Summer 2012, he worked as an Intern at Broadcom Corporation, Sunnyvale, CA. He is currently with Qualcomm Technologies Inc., Santa Clara, CA. His research interests include cooperative communications, applied signal processing, and radio resource management in wireless networks. 


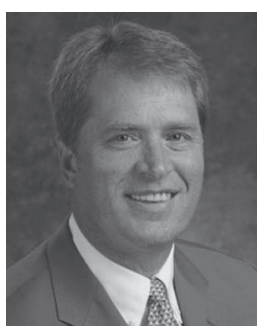

A. Lee Swindlehurst (S'83-M'84-SM'99-F'04) received the B.S. (summa cum laude) and M.S. degrees from Brigham Young University, Provo, Utah, in 1985 and 1986, respectively, and the Ph.D. degree from Stanford University, Stanford, CA, in 1991, all in electrical engineering.

From 1986 to 1990, he was employed at ESL, Inc., Sunnyvale, CA, where he was involved in the design of algorithms and architectures for several radar and sonar signal processing systems. From 1990 to 2007, he was on the faculty of the Department of Electrical and Computer Engineering, Brigham Young University, where he was a Full Professor and served as the Department Chair from 2003 to 2006. From 1996 to 1997, he held a joint appointment as a Visiting Scholar at both Uppsala University, Uppsala, Sweden, and at the Royal Institute of Technology, Stockholm, Sweden. From 2006 to 2007, he was on leave working as Vice President of Research for ArrayComm LLC,San Jose, CA. He is currently a Professor of electrical engineering and computer science with the University of California, Irvine, CA. His research interests include sensor array signal processing for radar and wireless communications, detection and estimation theory, and system identification. He has over 200 publications in these areas.

Dr. Swindlehurst is a past Secretary of the IEEE Signal Processing Society, a past Editor-in-Chief of the IEEE Journal OF SELECTED TOPICS IN SigNAL PROCESSING, and a past member of the Editorial Boards for the EURASIP Journal on Wireless Communications and Networking, IEEE SIGnal Processing Magazine, and the IEEE Transactions on Signal PROCESSING. He has received several paper awards, including the 2000 IEEE W. R. G. Baker Prize Paper Award, the 2006 and 2010 IEEE Signal Processing Society's Best Paper Awards, and the 2006 IEEE Communications Society Stephen O. Rice Prize in the Field of Communication Theory. He is also a co-author of a paper that received the IEEE Signal Processing Society Young Author Best Paper Award in 2001. 